\title{
Blind Channel Equalization with Colored Source Based on Constrained Optimization Methods
}

\author{
Yunhua Wang, ${ }^{1}$ Linda DeBrunner, ${ }^{2}$ Victor DeBrunner, ${ }^{2}$ and Dayong Zhou ${ }^{3}$ \\ ${ }^{1}$ Department of Electrical and Computer Engineering, Oklahoma University, Norman, OK 73072, USA \\ ${ }^{2}$ Department of Electrical and Computer Engineering, Florida State University, Tallahassee, FL 32306, USA \\ ${ }^{3}$ Cirrus Logic Inc., 2901 Via Fortuna, Austin, TX 78746, USA \\ Correspondence should be addressed to Dayong Zhou, dayong@ou.edu
}

Received 20 February 2008; Revised 23 June 2008; Accepted 11 September 2008

Recommended by Magnus Jansson

\begin{abstract}
Tsatsanis and $\mathrm{Xu}$ have applied the constrained minimum output variance (CMOV) principle to directly blind equalize a linear channel - a technique that has proven effective with white inputs. It is generally assumed in the literature that their CMOV method can also effectively equalize a linear channel with a colored source. In this paper, we prove that colored inputs will cause the equalizer to incorrectly converge due to inadequate constraints. We also introduce a new blind channel equalizer algorithm that is based on the CMOV principle, but with a different constraint that will correctly handle colored sources. Our proposed algorithm works for channels with either white or colored inputs and performs equivalently to the trained minimum mean-square error (MMSE) equalizer under high SNR. Thus, our proposed algorithm may be regarded as an extension of the CMOV algorithm proposed by Tsatsanis and $\mathrm{Xu}$. We also introduce several methods to improve the performance of our introduced algorithm in the low SNR condition. Simulation results show the superior performance of our proposed methods.
\end{abstract}

Copyright (c) 2008 Yunhua Wang et al. This is an open access article distributed under the Creative Commons Attribution License, which permits unrestricted use, distribution, and reproduction in any medium, provided the original work is properly cited.

\section{INTRODUCTION}

In digital communication, the multipath effect in a channel will subject the signal to intersymbol interference (ISI). The ISI will increase the symbol error rate (SER) at the receiver, sometimes making a correct estimation of the sent signal impossible. As a result, equalizers are required to remove the channel distortion. Roughly speaking, two kinds of equalizers in digital communication systems exist: data aided (trained) equalizers and blind equalizers. For data aided equalizers, a reference signal is required, increasing the data bandwidth. As a result, a blind equalizer is preferred in highspeed communication systems due to its potential to reduce the ISI without increasing the overhead costs.

Blind channel equalization relies solely on the channel output, with/without some a priori statistical knowledge of the input of the channel. Blind system equalization for a single input can be divided into two categories: single input single output (SISO) configurations, for example, the constant modulus algorithm (CMA), and single input multiple output (SIMO) configurations. Note that all SISO blind identification and equalization algorithms explicitly or implicitly exploit the high-order statistics of the input and output signals. As a result they all suffer from local minima or slow convergence $[1,2]$.

The SIMO configuration can be obtained from the exploitation of temporal (oversampling) or spatial (multiantenna) diversity of the received signal. The TXK algorithm developed by Tong et al. [3] first proved that the channel information could be blindly estimated using only secondorder statistics by exploiting the diversity. Different SIMO blind channel estimation and equalization algorithms have been proposed, such as the subchannel matching algorithm [4], the subspace algorithm [5], the linear prediction algorithm $[6,7]$, adaptive least square smoothing [8], and the outer product decomposition algorithm $[9,10]$ (some details about these and other algorithms can be found in [1] and its references). The popularity of SIMO, rather than SISO, blind channel identification and equalization comes from the fast convergence and efficient computation of these algorithms.

Most of the SIMO-based blind channel estimation and equalization methods assume that the channel input is white; as a result, the designed equalizers are sensitive to the color of the input. One could use a whitening filter 
to prewhiten the colored source before transmission; then use channel equalization for white sources to remove the channel effect; and then finally inverse filter to recover the original source. However, sometimes this complicated process may not be possible due to the inaccessibility of the input or the unavailability of the exact inverse filter; in any case, the prewhitening and inverse processes complicate the overall system required in this approach. Consequently, several researchers, such as López-Valcarce and Dasgupta and Afkhamie and Luo, have attempted to extend the TXK method to solve the colored input problem $([11,12]$, resp.), but the algorithms either entail many restrictions or have a large computational burden. Some SIMO-based blind channel equalization methods do not require assumptions regarding the input statistics, so they can be applied to systems with either white or colored inputs. For example, the subspace-based method introduced by Moulines et al. [5] could work for colored inputs. However, usually, the equalizer design requires a two-step procedure-the first step is to estimate the channel coefficients while the second step is to invert the channel effects using either zero forcing or an MMSE equalizer. Moreover, these methods do not exploit the input statistics - something that is already known to improve equalizer performance [11], though at a cost of increasing computational complexity.

Tsatsanis and $\mathrm{Xu}$ [13] proposed a direct blind equalization method by incorporating the constrained minimum output variance (CMOV), which is widely used in array signal processing. Based on their algorithm, the blind equalizer achieves a performance close to the trained MMSE equalizer for channels with white inputs at high SNR. The introduced Tsatsanis and Xu's (TX's) CMOV algorithm obtains the channel information from the noise subspace $[13,14]$. As a result, this algorithm can be regarded as a subspace method. However, unlike the subspace method discussed in [5], the TX's CMOV-based algorithm requires less computational complexity. Furthermore, using the CMOV principle, an adaptive blind channel equalization algorithm has been developed in [15]. However, the TX's CMOV algorithm does not work for colored input, though it is believed to work in this case; see, for example, $[1,13]$.

Colored sources may occur, for example, as a result of channel encoding. Under this situation, the knowledge of the encoding scheme alone will provide the required source statistics to the receiver [16]. In this work, we develop a new blind channel equalizer for channels with colored inputs based on the known source second-order statistics. Note that our developed method is different from both semiblind channel estimation which assumes additional knowledge of the symbol, and trained equalization which requires training sequences. By contrast, in our configuration, no training sequences are required, and only the second-order statistical information of the input is available to the receiver.

The contributions of this paper are two-fold. First, we point out and correct a widely-held misunderstanding about the previous developed CMOV-based channel equalization algorithm. Second, we extend the application of the CMOV principle by finding a new constraint and develop an efficient blind channel equalization method that works for both colored and white inputs. A part of these research results has been presented in [17]. In our notation, the superscripts $(\cdot)^{*},(\cdot)^{T}$, and $(\cdot)^{H}$ denote, respectively, the conjugate, the transpose, and the Hermitian transpose, and $E(\cdot)$ denotes expected value.

\section{PROBLEM DEFINITION}

Figure 1 shows the baseband representation of an SIMO data communication system with input $s(k)$. The left side of the figure represents the multichannels $h_{i}(k)$ with multiple measurements $x_{i}(k)$ while the right represents the multichannel equalizer $g_{i}(k)$ with output $y(k)$. The signals $n_{i}(k)$ are white noise. There are $p$ channels in Figure 1 SIMO configuration. The SIMO channel in vector form is

$$
\overline{\mathbf{x}}(k)=\sum_{i=0}^{q} \mathbf{h}(i) s(k-i)+\overline{\mathbf{n}}(k)
$$

with

$\mathbf{h}(i) \triangleq\left[\begin{array}{c}h_{1}(i) \\ \vdots \\ h_{p}(i)\end{array}\right], \quad \overline{\mathbf{x}}(k) \triangleq\left[\begin{array}{c}x_{1}(k) \\ \vdots \\ x_{p}(k)\end{array}\right], \quad \overline{\mathbf{n}}(k) \triangleq\left[\begin{array}{c}n_{1}(k) \\ \vdots \\ n_{p}(k)\end{array}\right]$.

Here, we denote the $i$ th term of the finite impulse response (FIR) of $j$ th channel as $h_{j}(i)$. We use the symbol $q$ to denote the order of the channel response. Equation (1) can be rewritten as

$$
\mathbf{x}(k)=\mathbf{T}(\mathbf{h}) \mathbf{s}(k)+\mathbf{n}(k)
$$

using the following definitions:

$$
\begin{aligned}
& \mathbf{s}(k) \triangleq\left[\begin{array}{c}
s(k) \\
\vdots \\
s(k-q-M)
\end{array}\right], \quad \mathbf{x}(k) \triangleq\left[\begin{array}{c}
\overline{\mathbf{x}}(k) \\
\vdots \\
\overline{\mathbf{x}}(k-M+1)
\end{array}\right], \\
& \mathbf{n}(k) \triangleq\left[\begin{array}{c}
\overline{\mathbf{n}}(k) \\
\vdots \\
\overline{\mathbf{n}}(k-M+1)
\end{array}\right] \\
& \mathbf{T}(\mathbf{h})=\underbrace{\left[\begin{array}{ccccccc}
\mathbf{h}(0) & \mathbf{h}(1) & \cdots & \mathbf{h}(q) & \mathbf{0} & \cdots & \mathbf{0} \\
\mathbf{0} & \mathbf{h}(0) & \ddots & \cdots & \mathbf{h}(q) & \ddots & \mathbf{0} \\
& & \ddots & \ddots & \ddots & \ddots & \vdots \\
\mathbf{0} & \mathbf{0} & \cdots & \mathbf{h}(0) & \mathbf{h}(1) & \cdots & \mathbf{h}(q)
\end{array}\right]}_{M+q} M p,
\end{aligned}
$$

where $M$ is the number of taps in the FIR equalizer $g_{i}(k)$ and $\mathbf{T}(\mathbf{h})$ is an $M p \times(M+q)$ block Toeplitz matrix. We denote the $i$ th column of $\mathbf{T}(\mathbf{h})$ by $\mathbf{h}_{i}$, that is,

$$
\mathbf{T}(\mathbf{h})=\left[\mathbf{h}_{1}, \mathbf{h}_{2}, \ldots, \mathbf{h}_{M+q}\right] .
$$

We define the channel coefficient vector as

$$
\mathbf{h}=\left[\begin{array}{lllll}
\mathbf{h}^{T}(q) & \mathbf{h}^{T}(q-1) & \cdots & \mathbf{h}^{T}(0)
\end{array}\right]^{T} .
$$




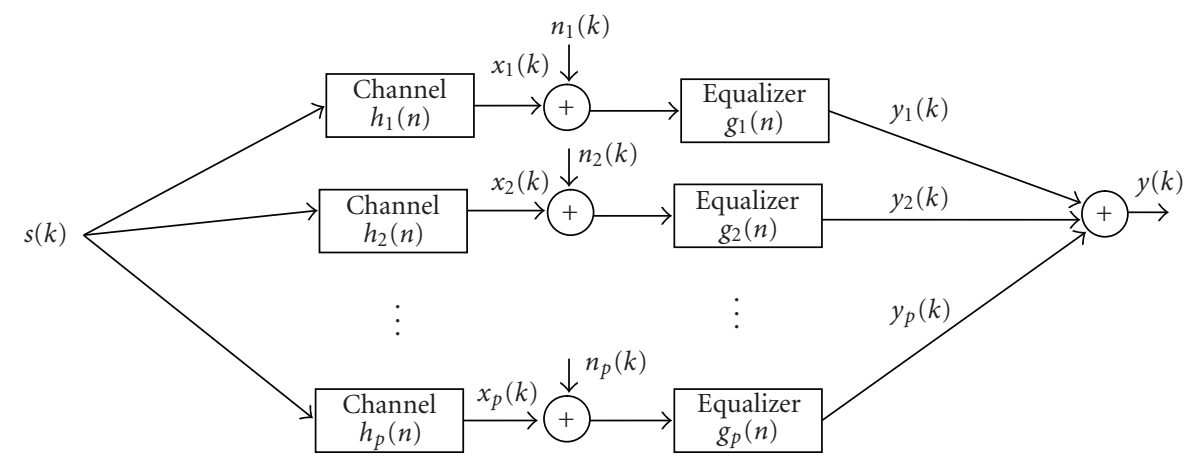

FIGURE 1: SIMO blind channel estimation and equalization.

Equations (5) and (6) will be useful in the following discussion. Our problem is to find $g_{i}(k)$ based on the following assumptions:

(AS1) the input $s(k)$ is unknown, but the second-order statistic $E\left(\mathbf{s}(k) \mathbf{s}^{H}(k)\right)$ is known and has full rank;

(AS2) $\mathbf{T}(\mathbf{h})$ has full column rank, that is, the $Z$-transforms of the $\mathbf{h}_{j}(1 \leq j \leq M+q)$ have no common zero;

(AS3) measurement noise $n_{i}(k)$ is independent and identically distributed (iid) zero mean noise with variance $\sigma_{n}^{2}$.

These are common assumptions in multichannel blind identification and equalization problems [5-10, 12]. For instance, note that (AS1) has been used in [11] to extend the TXK method for colored input.

In the same manner that we defined the vector structure in (3), we define the equalizer $\mathbf{g}$ as follows:

$$
\begin{aligned}
\overline{\mathbf{g}}(i) & \stackrel{\Delta}{=}\left[g_{1}(i), g_{2}(i), \ldots, g_{p}(i)\right]^{T}, \\
\mathbf{g} & \triangleq\left[\overline{\mathbf{g}}^{T}(0), \overline{\mathbf{g}}^{T}(1), \ldots, \overline{\mathbf{g}}^{T}(M-1)\right]^{T},
\end{aligned}
$$

where $g_{j}(i)$ denotes the $i$ th term of the $j$ th FIR equalizer. We want the output of the equalizer to be an undistorted version of the input, that is, we allow

$$
y(k)=\mathbf{g}^{H} \mathbf{x}(k)=\mathbf{g}^{H} \mathbf{T}(\mathbf{h}) \mathbf{s}(k)=s(k-d),
$$

where $d$ is some integer. For the trained MMSE equalizer [18]

$$
\mathbf{g}_{\mathrm{MMSE}}=\mathbf{R}_{x}^{-1} E[\mathbf{x}(k) s(k-d)]=\mathbf{R}_{x}^{-1} \mathbf{T}(\mathbf{h}) E\left[\mathbf{s}(k) s^{*}(k-d)\right],
$$

where $\mathbf{R}_{x}$ is the channel output covariance matrix

$$
\mathbf{R}_{x}=E\left\{\mathbf{x}(k) \mathbf{x}^{H}(k)\right\} .
$$

Note if the source is white, the autocorrelation of the source becomes a delta function. Therefore, the MMSE equalizer for white input becomes

$$
\mathbf{g}_{\text {MMSE }}=\mathbf{R}_{x}^{-1} \mathbf{h}_{d+1} .
$$

From (9), we see that to determine the MMSE equalizer, we must have the channel coefficient matrix and source signal second-order statistics. However, for our problem, the channel matrix $\mathbf{T}(\mathbf{h})$ is not available. We desire to find a blind equalizer $\mathbf{g}$ with performance close to $\mathbf{g}_{\text {MMSE }}$ that works for both white and colored inputs.

\section{ANALYSIS OF EXISTING CMOV METHOD}

Tsatsanis and $\mathrm{Xu}$ [13], borrowing from work in array signal processing, proposed a CMOV method which successfully solves the above problem when the input $s(n)$ is white and the SNR of the measured $x(n)$ is high. The equalizer is developed using the constrained optimization:

$$
\arg \min _{\mathbf{g}} E\left\{\|y(k)\|^{2}\right\}=\min _{\mathbf{g}} \mathbf{g}^{H} \mathbf{R}_{x} \mathbf{g} \quad \text { with } \mathbf{g}^{H} \mathbf{h}_{d+1}=1 \text {. }
$$

$\mathbf{h}_{d+1}$ is defined in (5). Using the method of Lagrange, the equalizer $\mathbf{g}$ is

$$
\mathbf{g}_{\mathrm{TX}}=\left(\mathbf{h}_{d+1}^{H} \mathbf{R}_{x}^{-1} \mathbf{h}_{d+1}\right)^{-1} \mathbf{R}_{x}^{-1} \mathbf{h}_{d+1} .
$$

Note that for a white input, the equalizer in (13) only has an amplitude difference from an optimum MMSE equalizer in (11). We can obtain the minimum output variance which is

$$
V_{\min }=\left(\mathbf{h}_{d+1}^{H} \mathbf{R}_{x}^{-1} \mathbf{h}_{d+1}\right)^{-1}
$$

However in blind channel equalization, the channel information $\mathbf{h}_{d+1}$ is unknown. Tsatsanis and $\mathrm{Xu}$ resorted to the Capon $\mathrm{max} / \mathrm{min}$ approach [19] to estimate the channel response $\mathbf{h}$. This approach may be succinctly described. Define the structure matrix $\mathbf{C}_{d+1}$

$$
\mathbf{C}_{d+1}=\left[\begin{array}{lll}
\mathbf{0}_{p(d-q) \times p(q+1)} & \mathbf{I}_{p(q+1) \times p(q+1)} & \mathbf{0}_{p(M-d-1) \times p(q+1)}
\end{array}\right]^{H}
$$

and channel coefficients vector $\mathbf{h}$ as in (6) so that

$$
\mathbf{h}_{d+1}=\mathbf{C}_{d+1} \mathbf{h}
$$

for $M \geq d+1 \geq q+1$. The estimated $\mathbf{h}$ is then obtained by maximizing the minimum output variance in (14), that is,

$$
\begin{aligned}
\widehat{\mathbf{h}} & =\arg \max _{\mathbf{h}} \frac{\mathbf{h}^{H} \mathbf{C}_{d+1}^{H} \mathbf{C}_{d+1} \mathbf{h}}{\mathbf{h}^{H} \mathbf{C}_{d+1}^{H} \mathbf{R}_{x}^{-1} \mathbf{C}_{d+1} \mathbf{h}} \\
& =\arg \min _{\mathbf{h}} \frac{\mathbf{h}^{H} \mathbf{C}_{d+1}^{H} \mathbf{R}_{x}^{-1} \mathbf{C}_{d+1} \mathbf{h}}{\mathbf{h}^{H} \mathbf{h}}
\end{aligned}
$$


Of course, the solution is the eigenvector corresponding to the minimum eigenvalue of $\mathbf{C}_{d+1}^{H} \mathbf{R}_{x}^{-1} \mathbf{C}_{d+1}$. The proof in [13] shows that

$$
\widehat{\mathbf{h}}=\frac{\mathbf{h}}{\|\mathbf{h}\|} \quad \text { as } \sigma_{n}^{2} \longrightarrow 0 .
$$

Using this estimated $\hat{\mathbf{h}}$, one can now compute the CMOV equalizer directly using (13). Note that the algorithm developed by Tsatsanis and $\mathrm{Xu}$ requires that the order of the equalizer must be above $3(q+1)$, and " $d$ is not allowed to take any of the first or last $2 q$ allowable lag $[s]$." These restrictions ensure that (18) holds.

It is believed that the above "are not sensitive to the color of the input" [13]. However, our simulations (refer to Section 7) show that the algorithm fails to generate a correct equalizer for a channel with colored input. Since the estimation of $\hat{\mathbf{h}}$ and the proof of (18) do not require white input, the estimation of the channel will not be affected by colored inputs. However, the very basis of the constrained optimization (12) will generate a biased equalizer in this case, which means the equalizer calculated by (13) cannot eliminate the channel effect for nonwhite inputs. The reason for the failure of the $\mathrm{CMOV}$ method is its inadequate constraints.

These inadequacies can be illustrated using the overall channel response model of Figure 1. The combined response of the $p$ channels and $p$ equalizers can be regarded as an SISO FIR filter $f(n)$ with order $M+q$. We want the overall response of the channel and equalizer, $f(n)$, to be only delay, and so only one coefficient of $f(n)$ can be nonzero. The coefficient vector of $f(n)$ is

$$
\mathbf{f}=\mathbf{g}^{H} \mathbf{T}(\mathbf{h})=\left[\mathbf{g}^{H} \mathbf{h}_{0}, \mathbf{g}^{H} \mathbf{h}_{1}, \ldots, \mathbf{g}^{H} \mathbf{h}_{d+1}, \ldots, \mathbf{g}^{H} \mathbf{h}_{M+q}\right] .
$$

The variance of the output of this FIR filter is

$$
\begin{aligned}
\gamma_{y}(0) & =\sum_{l=0}^{M+q} \sum_{m=0}^{M+q-1} \gamma_{s}(m-l) f^{H}(m) \\
& =\mathbf{f R}_{s} \mathbf{f}^{H},
\end{aligned}
$$

where $r_{s}(n)=E\left[s(k) s^{*}(k-n)\right]$ and $\mathbf{R}_{s}$ is the autocorrelation matrix of input signal $s(k)$. For white inputs,

$$
r_{y}(0)=r_{s}(0) \sum_{n=0}^{M+q-1}\|f(n)\|^{2} .
$$

Using the constraint $f(d)=\mathbf{g}^{T} \mathbf{h}_{d+1}=1 \neq 0$, the minimum output variance is achieved when all coefficients in $\mathbf{f}$ are zero except $f(d)$, that is, the filter $f(n)$ is a delay of $d$ samples. However, for colored inputs, minimizing $r_{y}(0)$ with the constraint $\mathbf{g}^{T} \mathbf{h}_{d+1}=1$ cannot guarantee that $f(n)$ will converge to a pure delay. Actually, the overall response $f(n)$ will force the frequency component of $y(n)$ to be the one's complement of the input signal $s(n)$, which is easily obtained by analyzing $\mathbf{g}_{\mathrm{TX}}$ in (13) [20].

\section{DEVELOPMENT OF OUR NEW CMOV ALGORITHM}

From our previous analysis, we find that the TX's CMOV method cannot correctly equalize a linear channel for colored input due to the inadequate constraint that causes the equalizer to converge to a biased value. To find a good equalizer based on the CMOV method is then to find an efficient constraint that forces the overall response $f(n)$ in (19) to be a simple delay. In this section, we first find this efficient constraint based on the overall response analyses in the previous section. Then we develop a new equalization algorithm based on the CMOV method that successfully removes the channel effects.

\subsection{An efficient constraint for colored input}

In CMOV-based equalization methods, the constraint plays a very important role. An efficient constraint should not only prevent the signal from being eliminated, but it should also guarantee the removal of the ISI. For our purposes, we define the efficient constraint mathematically in the following.

Definition 1. Consider the constraint $\mathbf{f} \gamma=\mathbf{1}$, where $\gamma$ is a vector of length $M+q$. If, using this constraint, the solution of $\arg \min _{\mathbf{f}} \mathbf{f R}_{s} \mathbf{f}^{H}$ is

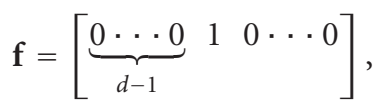

then we say this constraint is an efficient constraint.

In this definition, vector $\mathbf{f}$ is the coefficient vector of the overall channel response defined in (19). The vector $y$ can be found using Lagrange multipliers. We first define a cost function

$$
E \frac{1}{2} \mathbf{f R}_{s} \mathbf{f}^{H}+\lambda\left(1-\mathbf{f} \boldsymbol{\gamma}^{H}\right) .
$$

Differentiating the right side of (23) with respect to $f$, the minimum achieved at $\partial E / \partial \mathbf{f}=0$ yields

$$
\begin{gathered}
\frac{\partial E}{\partial \mathbf{f}}=\mathbf{R}_{s} \mathbf{f}^{H}-\lambda \boldsymbol{\gamma}=0, \\
\boldsymbol{\gamma}=\frac{1}{\lambda} \mathbf{R}_{s} \mathbf{f}^{H} .
\end{gathered}
$$

Note that $\mathbf{R}_{s}$ is invertible based on (AS1). Considering

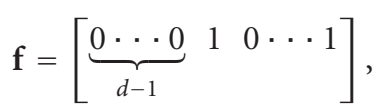

we find that $\gamma$ should be the $d$ th column of the input correlation matrix, that is,

$$
\begin{aligned}
y & =\frac{1}{\lambda} E\left[\mathbf{s}(k) s^{*}(k-d)\right] \\
& =\frac{1}{\lambda}\left[r_{s}(d), r_{s}(d-1), \ldots, r_{s}(0), \ldots, r_{s}(M+q-d-1)\right]^{H}
\end{aligned}
$$

which of course is the correlation between the delayed input and the input vector. Based on (26) and Definition 1, we 
can have the efficient constraint $\mathbf{f} \boldsymbol{\gamma}=\mathbf{1}$, so that the overall response $f(n)$ can be guaranteed to be only a delay. As a result, using this constraint, we can successfully reduce the ISI caused by a channel. Note that we do not consider the measurement noise in finding the efficient constraint. However, we prove next that the blind equalizer we develop in this paper is an MMSE-like equalizer instead of zero force (ZF) equalizer.

\subsection{New CMOV-based blind channel equalizer}

Based on the efficient constraint developed in the previous subsection, we first prove the following proposition before we introduce our new CMOV-based blind channel equalization method.

Proposition 1. If $\mathbf{g}^{H}=\arg \min _{\mathbf{g}^{H}} E\left\{\|y(k)\|^{2}\right\}=$ $\arg \min _{\mathbf{g}^{H}} \mathbf{g}^{H} \mathbf{R}_{x} \mathbf{g}$ with $\mathbf{g}^{H} \mathbf{T}(\mathbf{h}) \boldsymbol{\gamma}=1$, then this solution differs from the MMSE equalizer only by a scalar factor gain.

Proof. This is a constrained optimization problem, which can also be solved using Lagrange multipliers. First define the cost function

$$
J=\frac{1}{2} \mathbf{g}^{H} \mathbf{R}_{x} \mathbf{g}+\lambda\left(1-\mathbf{g}^{H} \mathbf{T}(\mathbf{h}) \boldsymbol{\gamma}\right) .
$$

Again, we use $\lambda$ as the Lagrange multiplier. Minimizing the cost function yields

$$
\begin{aligned}
\lambda & =\left(\boldsymbol{\gamma}^{H} \mathbf{T}(\mathbf{h})^{H} \mathbf{R}_{x}^{-1} \mathbf{T}(\mathbf{h}) \boldsymbol{\gamma}\right)^{-1}, \\
\mathbf{g}^{H} & =\frac{\mathbf{R}_{x}^{-1} \mathbf{T}(\mathbf{h}) \boldsymbol{\gamma}}{\boldsymbol{\gamma}^{H} \mathbf{T}(\mathbf{h})^{H} \mathbf{R}_{x}^{-1} \mathbf{T}(\mathbf{h}) \boldsymbol{\gamma}} .
\end{aligned}
$$

Comparing (29) with (9), we see only a scalar factor difference between $\mathbf{g}^{H}$ and the MMSE equalizer.

Note that the similar CMOV concept has been applied in multiuser detection and array signal processing [21]. Proposition 1 provides the theoretical background of our new algorithm. However, as with the method in TX [13], this constrained optimization requires channel information that is not available. In order to estimate the channel information, we need to resort to the Capon max/min method.

The minimum output variance is obtained when $\mathbf{g}^{H}$ takes the value in (29)

$$
V_{\min }\left(\mathbf{g}^{H}\right)=\left(\gamma^{H} \mathbf{T}(\mathbf{h})^{H} \mathbf{R}_{x}^{-1} \mathbf{T}(\mathbf{h}) \gamma\right)^{-1} .
$$

Based on the Capon max/min method, we can find the channel information by maximizing the minimum output variance $V_{\min }\left(\mathbf{g}^{H}\right)$. However, it is not easy to directly apply the $\max / \mathrm{min}$ method. We define an extension of the vector $\gamma$

$$
\begin{aligned}
& \gamma_{\mathrm{ext}}= \\
& {[\underbrace{0, \ldots, 0}_{p-1}, \gamma(M+q), \underbrace{0, \ldots, 0}_{p-1}, \gamma(M+q-1), \ldots, \gamma(1), \underbrace{0, \ldots, 0}_{p-1}]^{H} .}
\end{aligned}
$$

Note that this extended vector is constructed by reversing the vector $\gamma$ and interspersing $p-1$ zeros between every element.
Then we construct the $p M \times p(q+1)$ Toeplitz matrix $\mathbf{T}\left(\boldsymbol{\gamma}_{\text {ext }}\right)$ of this $\gamma_{\text {ext }}$ as

$$
\mathbf{T}\left(\gamma_{\mathrm{ext}}\right)=\left[\begin{array}{c}
\gamma_{\mathrm{ext}}^{H}(p M: p M+p q+p-1) \\
\gamma_{\mathrm{ext}}^{H}(p M-1: p M+p q+p-2) \\
\vdots \\
\gamma_{\mathrm{ext}}^{H}(1: p(q+1))
\end{array}\right] .
$$

Lemma 1. The following relation holds

$$
\mathbf{T}(\mathbf{h}) \boldsymbol{\gamma}=\mathbf{T}\left(\boldsymbol{\gamma}_{\mathrm{ext}}\right) \mathbf{h} .
$$

This lemma can be proven by direct substitution or using a method based on the Z-transform [5]. One anonymous reviewer also pointed out that this is commutative property of convolution in a matrix formulation. Using (31) and Lemma 1, we rewrite (30) as

$$
V_{\min }\left(\mathbf{g}^{H}\right)=\left(\mathbf{h}^{H} \mathbf{T}\left(\gamma_{\mathrm{ext}}\right)^{H} \mathbf{R}_{x}^{-1} \mathbf{T}\left(\boldsymbol{\gamma}_{\mathrm{ext}}\right) \mathbf{h}\right)^{-1} .
$$

At this point, we can apply the Capon max/min principle to estimate $\mathbf{h}$

$$
\begin{aligned}
\overline{\mathbf{h}}_{\text {capon }} & =\arg \max _{\mathbf{h}}\left(\mathbf{h}^{H} \mathbf{T}\left(\boldsymbol{\gamma}_{\text {ext }}\right){ }^{H} \mathbf{R}_{x}^{-1} \mathbf{T}\left(\boldsymbol{\gamma}_{\text {ext }}\right) \mathbf{h}\right)^{-1} \\
& =\arg \min _{\mathbf{h}} \mathbf{h}^{H} \mathbf{T}\left(\boldsymbol{\gamma}_{\text {ext }}\right){ }^{H} \mathbf{R}_{x}^{-1} \mathbf{T}\left(\boldsymbol{\gamma}_{\text {ext }}\right) \mathbf{h} .
\end{aligned}
$$

We see that $\overline{\mathbf{h}}_{\text {capon }}$ is equal to the eigenvector corresponding to the minimum eigenvalue of $\mathbf{T}\left(\boldsymbol{\gamma}_{\mathrm{ext}}\right)^{H} \mathbf{R}_{x}^{-1} \mathbf{T}\left(\boldsymbol{\gamma}_{\mathrm{ext}}\right)$. Thus, our algorithm for blind channel equalizer design can be summarized in the following steps:

(1) obtain the source statistics $\gamma_{\mathrm{ext}}$ and $\mathbf{R}_{x}^{-1}$;

(2) construct $\mathbf{T}\left(\boldsymbol{\gamma}_{\mathrm{ext}}\right)$ and $\mathbf{A}=\mathbf{T}\left(\boldsymbol{\gamma}_{\mathrm{ext}}\right)^{H} \mathbf{R}_{x}^{-1} \mathbf{T}\left(\boldsymbol{\gamma}_{\mathrm{ext}}\right)$;

(3) estimate the channel $\overline{\mathbf{h}}_{\text {capon }}$ coefficient by finding the minimum eigenvalue and corresponding eigenvector of A;

(4) find the equalizer $\overrightarrow{\mathbf{g}}^{H}=\mathbf{R}_{x}^{-1} \mathbf{T}\left(\boldsymbol{\gamma}_{\mathrm{ext}}\right) \overline{\mathbf{h}}_{\text {capon; }}$;

(5) remove the phase ambiguity which is inherent to all SOS-based method.

\section{CONVERGENCE ANALYSIS}

The main difference between our proposed CMOV algorithm and TX's CMOV algorithm is the different constraint. We already proved that TX's CMOV algorithm cannot generate an adequate equalizer for colored input due to its inadequate constraint. In this section, we will see that our proposed blind equalizer converges to trained MMSE equalizer for high SNR systems following the similar convergence analyses approach in [13].

As shown in (29) and step (4) of our algorithm, if the estimated $\overline{\mathbf{h}}_{\text {capon }}$ is equal to the correct channel coefficient vector $\mathbf{h}$, then the blind equalizer differs from the MMSE 
equalizer only by a scale factor. The scale can be corrected by comparing the power of the equalizer output and the system input. Consequently, our developed equalizer will have equivalent performance to the trained MMSE equalizer. As a result, how well the estimated channel coefficients vector relates to the true vector will determine the performance of the proposed equalizer. To see this, we need to prove the following proposition.

Proposition 2. If the order of the equalizer $M \geq(p+q p+$ $q) /(p-1)$ and the number of delays $d$ satisfy the constraint $M-$ $(p+q) /(p-1) \geq d \geq p q /(p-1)$, as the SNR $\rightarrow \infty$, the $\overline{\mathbf{h}}_{\text {capon }}$ estimated in (35) differs from the true channel coefficients by a phase and scale factor, that is,

$$
\overline{\mathbf{h}}_{\text {capon }}=\frac{e^{j \theta} \mathbf{h}}{\|\mathbf{h}\|} \quad \text { as } \sigma_{n}^{2} \longrightarrow 0
$$

Proof. We follow the same steps as in [13], that is, we first prove $\mathbf{h}$ is a solution of $\arg \min _{\mathbf{h}} \mathbf{h}^{H} \mathbf{T}\left(\boldsymbol{\gamma}_{\text {ext }}\right)^{H} \mathbf{R}_{x}^{-1} \mathbf{T}\left(\boldsymbol{\gamma}_{\text {ext }}\right) \mathbf{h}$ as $\sigma_{n}^{2} \rightarrow 0$. We then prove this solution is unique within a scalar constant under the condition given by Proposition 2 .

Step 1. We first write the eigen-decomposition of $\mathbf{R}_{x}$ :

$$
\mathbf{R}_{x}=\left[\begin{array}{ll}
\mathbf{V}_{s} & \mathbf{V}_{n}
\end{array}\right]\left[\begin{array}{cc}
\boldsymbol{\Lambda}_{s} & \mathbf{0} \\
\mathbf{0} & \mathbf{0}
\end{array}\right]\left[\begin{array}{c}
\mathbf{V}_{s}^{H} \\
\mathbf{V}_{n}^{H}
\end{array}\right]+\sigma_{n}^{2} \mathbf{I},
$$

where $\boldsymbol{\Lambda}_{s}=\operatorname{diag}\left\{\lambda_{1}, \ldots, \lambda_{M+q}\right\}$ and where $\mathbf{V}_{s}$ and $\mathbf{V}_{n}$ represent the signal and noise subspaces, respectively. It has been proved in $[13$, equation (32)] that

$$
\sigma_{n}^{2} \mathbf{C}_{d+1}^{H} \mathbf{R}_{x}^{-1} \mathbf{C}_{d+1} \longrightarrow \mathbf{C}_{d+1}^{H} \mathbf{V}_{n} \mathbf{V}_{n}^{H} \mathbf{C}_{d+1}
$$

as $\sigma_{n}^{2} \rightarrow 0$. Similarly we can have

$$
\sigma_{n}^{2} \mathbf{A}=\sigma_{n}^{2} \mathbf{T}\left(\boldsymbol{\gamma}_{\text {ext }}\right){ }^{H} \mathbf{R}_{x}^{-1} \mathbf{T}\left(\boldsymbol{\gamma}_{\text {ext }}\right) \longrightarrow \mathbf{T}\left(\boldsymbol{\gamma}_{\text {ext }}\right)^{H} \mathbf{V}_{n} \mathbf{V}_{n}^{H} \mathbf{T}\left(\boldsymbol{\gamma}_{\text {ext }}\right)
$$

as $\sigma_{n}^{2} \rightarrow 0$. So, the eigenvectors of $\mathbf{A}$ form the noise subspace. Because the signal subspace $\mathbf{V}_{s}$ is orthogonal to the noise subspace and $\mathbf{T}\left(\boldsymbol{\gamma}_{\text {ext }}\right) \mathbf{h}=\mathbf{T}(\mathbf{h}) \boldsymbol{\gamma} \in \mathbf{V}_{s}$, we get $\mathbf{V}_{n}^{H} \mathbf{T}\left(\gamma_{\text {ext }}\right) \mathbf{h}=0$. Consequently, we find that $\mathbf{h}$ is a solution of $\arg \min _{\mathbf{h}} \mathbf{h}^{H} \mathbf{T}\left(\boldsymbol{\gamma}_{\text {ext }}\right)^{H} \mathbf{R}_{x}^{-1} \mathbf{T}\left(\boldsymbol{\gamma}_{\text {ext }}\right) \mathbf{h}$.

Step 2. Prove under the above conditions that $\mathbf{h}$ is the only solution of $\arg \min _{\mathbf{h}} \mathbf{h}^{H} \mathbf{T}\left(\boldsymbol{\gamma}_{\text {ext }}\right)^{H} \mathbf{R}_{x}^{-1} \mathbf{T}\left(\boldsymbol{\gamma}_{\text {ext }}\right) \mathbf{h}$. It is equivalent to prove that there is no other vector $\mathbf{h}^{\prime}$ with $\mathbf{h}^{\prime} \neq c \mathbf{h}(c$ a complex constant), so that $\mathbf{T}\left(\boldsymbol{\gamma}_{\text {ext }}\right) \mathbf{h}^{\prime} \in \mathbf{V}_{s}$.

This step is proven by contradiction. Assume that we have $\mathbf{T}\left(\boldsymbol{\gamma}_{\mathrm{ext}}\right) \mathbf{h}^{\prime} \in \mathbf{V}_{s}$. Then

$$
\mathbf{T}\left(\gamma_{\text {ext }}\right) \mathbf{h}^{\prime}=\mathbf{T}\left(\mathbf{h}^{\prime}\right) \gamma \in \mathbf{V}_{s}
$$

Consequently,

$$
\mathbf{T}\left(\mathbf{h}^{\prime}\right) \boldsymbol{\gamma}=\mathrm{T}(\mathbf{h}) \boldsymbol{\theta}
$$

where $\boldsymbol{\theta}$ is a $(M+q) \times 1$ vector and the elements in $\boldsymbol{\theta}$ are unknown constants. We need to prove that (41) has only one set of solutions $\boldsymbol{\theta}$ and $\mathbf{T}\left(\mathbf{h}^{\prime}\right)$. Equation (41) is a set of linear equations with $M+q+p(q+1)$ unknowns and $M p$ equations when every component of $\boldsymbol{\gamma}$ is nonzero. Considering $\mathbf{T}(\mathbf{h})$ has full column rank, we only need the number of equations to be greater than or equal to the number of unknowns to ensure there is a unique solution. Consequently, we find that the order of the blind nonlinear equalizer is

$$
M \geq \frac{p+q p+q}{p-1} .
$$

This condition shows that increasing the number of channels will reduce the requirements on the order of the equalizer. Furthermore, to successfully equalize the channel, we need at least two channels, that is, $p \geq 2$.

When the input is white noise, the ideal $\gamma$ has only one nonzero component. Combining this fact with the Toeplitz structure of $\mathbf{T}(\mathbf{h})$ and $\mathbf{T}\left(\mathbf{h}^{\prime}\right)$ in (41), we see that the number of equations is $\left(d_{\min }+1\right) p$ and the number of unknowns is $p(q+1)+d$ for the minimum delay $d_{\min }$. Also, the number of equations is $\left(M-d_{\max }+q\right) p$ and the number of unknowns is $p(q+1)+M+q-d_{\max }$ for the maximum delay $d_{\max }$. Thus, the delay range is

$$
M-\frac{p+q}{p-1} \geq d \geq \frac{p q}{p-1} .
$$

Under these conditions, we can have one and only one solution, that is, $\mathbf{h}^{\prime}=\mathbf{h}$. So $\mathbf{h}$ is the only solution under our assumptions. For colored input, if $\gamma$ does not meet either of the above two conditions, the delay $d$ will take a wider range of values than that in (43) depending on the value of $\gamma$. As a result, the given condition in (43) is a sufficient condition for colored inputs.

Proposition 2 shows that our proposed method asymptotically converges to the MMSE equalizer as the SNR increases and that the proposed method has some constraints on the equalizer order and the number of delays. It is interesting to see that, although based on different constraints, both our method and the TX's CMOV algorithm can be used to estimate the channel coefficients. This is because the underlying estimation implicitly makes use of the subspace method, which is insensitive to colored inputs [5]. However, we will see that our proposed algorithm outperforms TX's CMOV in the estimation of the channel coefficients due to the use of the input statistics in our simulations. For white input and long data sequences, our algorithm reduces to the TX's CMOV algorithm, because under this condition,

$$
\gamma=\left[\begin{array}{llll}
\underbrace{0 \cdots 0}_{d-1} & 1 & 0 \cdots 0
\end{array}\right],
$$

and so our constraint, $\mathbf{g}^{H} \mathbf{T}(\mathbf{h}) \boldsymbol{\gamma}=1$, is equivalent to the CMOV constraint $\mathbf{g}^{H} \mathbf{h}_{d+1}=1$. As a result, our developed algorithm is an extension of TX's COMV algorithm. 


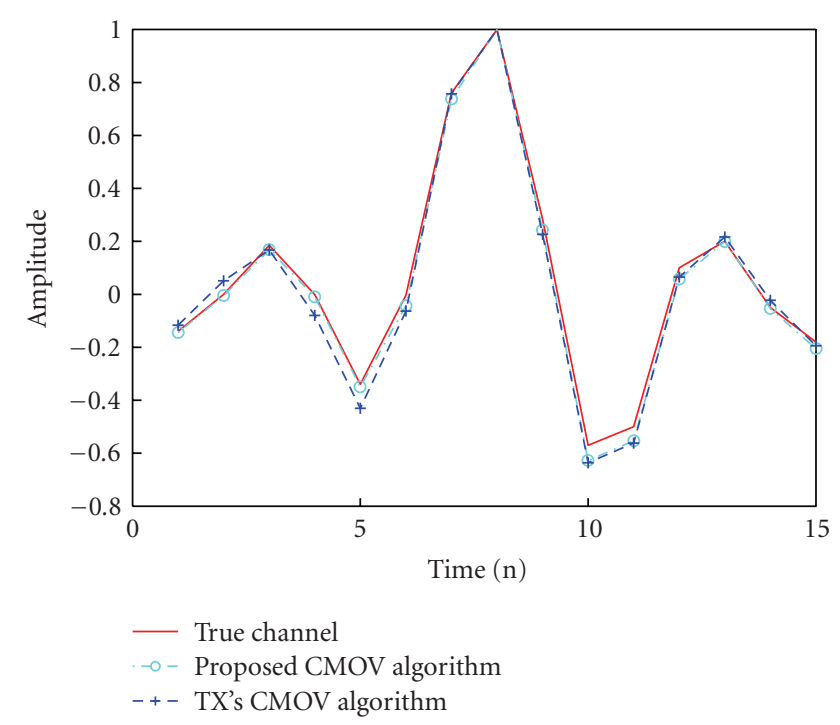

FIGURE 2: The channel estimation for colored input.

\section{FURTHER IMPROVEMENT}

We showed in Section 5 that the performance of our proposed blind channel equalizer will converge asymptotically to the MMSE equalizer as $\sigma_{n}^{2} \rightarrow 0$. However, for low SNR, the proposed algorithm will perform poorly due to the rough approximation used in (39). There are methods available to improve the SNR of the autocorrelation matrix. One is the matrix denoising method introduced by Moulines et al. [5]; another is the power of $\mathrm{R}$ (POR) method developed by $\mathrm{Xu}$ et al. [14, 22]. Here, we borrow these ideas and propose several extensions to our method that improve its performance in the low SNR situation without performance analyses. Application of each of these methods will only alter Step 2 in our proposed algorithm, that is, the calculation of matrix A. Instead of calculating $\mathbf{A}$ using $\mathbf{T}\left(\gamma_{\text {ext }}\right){ }^{H} \mathbf{R}_{x}^{-1} \mathbf{T}\left(\boldsymbol{\gamma}_{\text {ext }}\right)$, we provide three alternative methods to calculate $\mathbf{A}$ in this case as follows.

\section{(1) Matrix denoising method}

$$
\mathbf{A}=\mathbf{T}\left(\gamma_{\mathrm{ext}}\right)^{H}\left[\mathbf{R}_{x}-\left(\lambda_{\min }-\delta\right) \mathbf{I}\right]^{-1} \mathbf{T}\left(\gamma_{\mathrm{ext}}\right),
$$

where $\lambda_{\text {min }}$ is the minimum eigenvalue of $\mathbf{R}_{x}$ and $\delta$ is a small positive constant. This method is a straightforward extension of the matrix denoising method in [5].

\section{(2) POR method}

$$
\mathbf{A}=\mathbf{T}\left(\boldsymbol{\gamma}_{\mathrm{ext}}\right){ }^{H} \mathbf{R}_{x}^{-m} \mathbf{T}\left(\boldsymbol{\gamma}_{\mathrm{ext}}\right),
$$

where $m$ is a constant integer and $m \geq 1$. Note that although this POR method applies the same principle as the method in both $[14,22]$, this POR method is different from that POR method. As for the TX's CMOV algorithm, the POR method in [14] will not work for a channel with colored inputs.

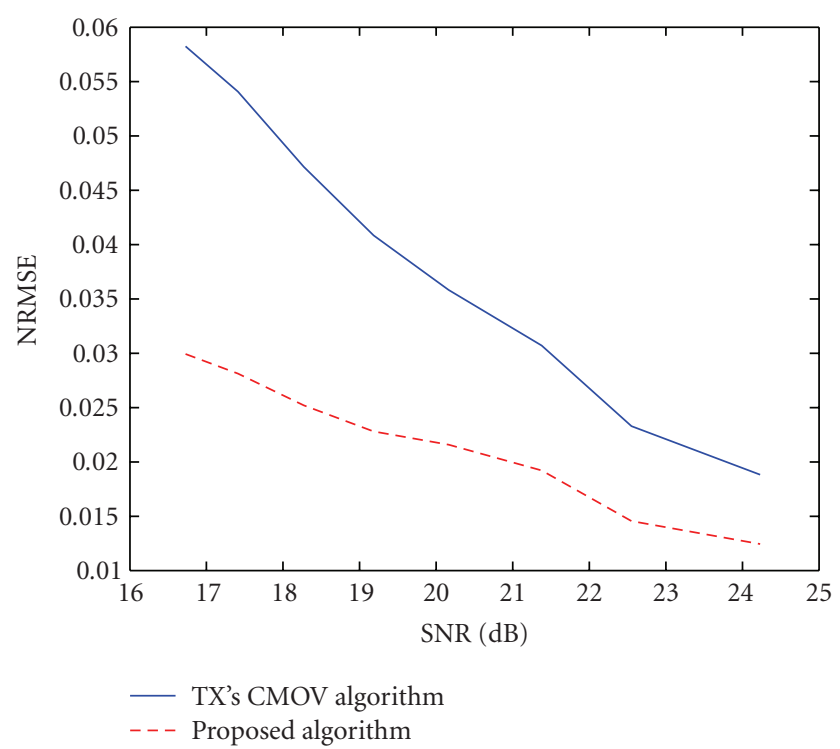

FIGURE 3: SNR and channel estimation NRMSE for colored inputs.

\section{(3) Hybrid method}

Combine the denoising and the POR methods so that the matrix A can be calculated using

$$
\mathbf{A}=\mathbf{T}\left(\boldsymbol{\gamma}_{\mathrm{ext}}\right)^{H}\left[\mathbf{R}_{x}-\left(\lambda_{\mathrm{min}}-\delta\right) \mathbf{I}\right]^{-m} \mathbf{T}\left(\boldsymbol{\gamma}_{\mathrm{ext}}\right) .
$$

We can see that these improvements are achieved at the cost of increasing the computational complexity.

\section{SIMULATIONS}

We simulate an SIMO communication system as shown in Figure 1. The FIR channel of order 15 is modeled by $g(t)=$ $c(t)-0.7 c(t-T / 3)$, where $c(t)$ is a raised-cosine pulse limited in $6 \mathrm{~T}$ with roll-off factor 0.10 and with an oversampling factor 3 , that is, $p=3$ in Figure 1 .

\subsection{Compare our method to previous CMOV methods}

In this simulation, the input $s(k)$ is generated by filtering an .id 4-PAM signal with a causal FIR filter whose impulse response coefficient vector is $\left[\begin{array}{llll}1 & -0.3 & 0.14 & 0.12\end{array}\right]$ to generate colored source. Please note, in practical application, the colored sources may occur, for example, as a result of channel encoding. We implement both our algorithm and TX's CMOV algorithm to equalize the linear channels. In the implementation of our algorithm, we only use the autocorrelation of the source, but not the FIR filter coefficients. The order of the equalizers is 16. Figure 2 shows the channel identification result at $\mathrm{SNR}=18 \mathrm{~dB}$ where the number of delays is equal to 8 and the data length equals 6000. We find that both algorithms identify the channel coefficients, though our method identifies the channel better than does TX's CMOV method in [13] because we make use of the input statistics. In Figure 3, we show an average of 50 runs of the relationship between the SNR and the normalized 


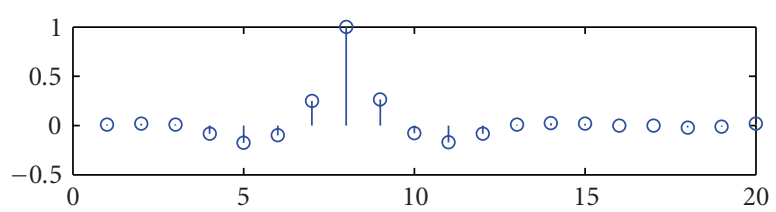

(a)

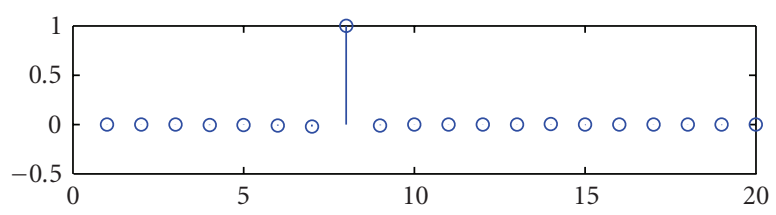

(b)

Figure 4: The overall response $f(n)$ of the channel and equalizer for colored input (a) based on TX's CMOV algorithm, (b) Proposed algorithm.

root mean-square error (NRMSE) of the estimated channel parameters, which is defined as [11]:

$$
\mathrm{NRMSE}=\sqrt{\frac{1}{M q} \sum_{n=0}^{M q-1}\left|\hat{\mathbf{h}}_{\text {capon }}(n)-\overline{\mathbf{h}}(n)\right|^{2}}
$$

We notice that as the SNR decreases, the improvement of our method over that of the TX CMOV algorithm is more pronounced, as shown in Figure 3. In Figure 4, we show an average of 50 runs of the overall response $f(n)$ defined in (17) at the SNR of $26 \mathrm{~dB}$, from which we can see that our algorithm successfully equalizes the channel distortion while the TX's CMOV method fails.

\subsection{SER performance comparison}

The previous simulations demonstrate that our proposed algorithm can successfully equalize linear channels for both colored and white inputs at relatively high SNR. The superior performances of the López-Valcarce algorithm [11] over other blind linear channel equalization algorithms for colored inputs, such as the subspace algorithm introduced by Moulines et al. [5] and the algorithm introduced by Afkhamie and Luo [12], have been demonstrated in [11]. As a result, in this simulation, we only compare our proposed algorithms with the López-Valcarce algorithm [11]. As we discussed earlier, the proposed algorithm performs poorly at low SNR, so we also implement the improved algorithms discussed in Section 6. In this simulation, the 3 linear channels possess the same coefficients as before. The inputs are the 4-QAM constellation, generated by the same rule as in [11], that is,

$$
s(n)=\left\{\begin{array}{ll}
-1+j & \text { if }\left(b_{n} b_{n-1}\right)=\left(\begin{array}{ll}
0 & 0
\end{array}\right) \\
+1+j & \text { if }\left(b_{n} b_{n-1}\right)=\left(\begin{array}{ll}
0 & 1
\end{array}\right) \\
-1-j & \text { if }\left(b_{n} b_{n-1}\right)=\left(\begin{array}{ll}
1 & 0
\end{array}\right) \\
+1+j & \text { if }\left(b_{n} b_{n-1}\right)=\left(\begin{array}{ll}
1 & 1
\end{array}\right)
\end{array} .\right.
$$

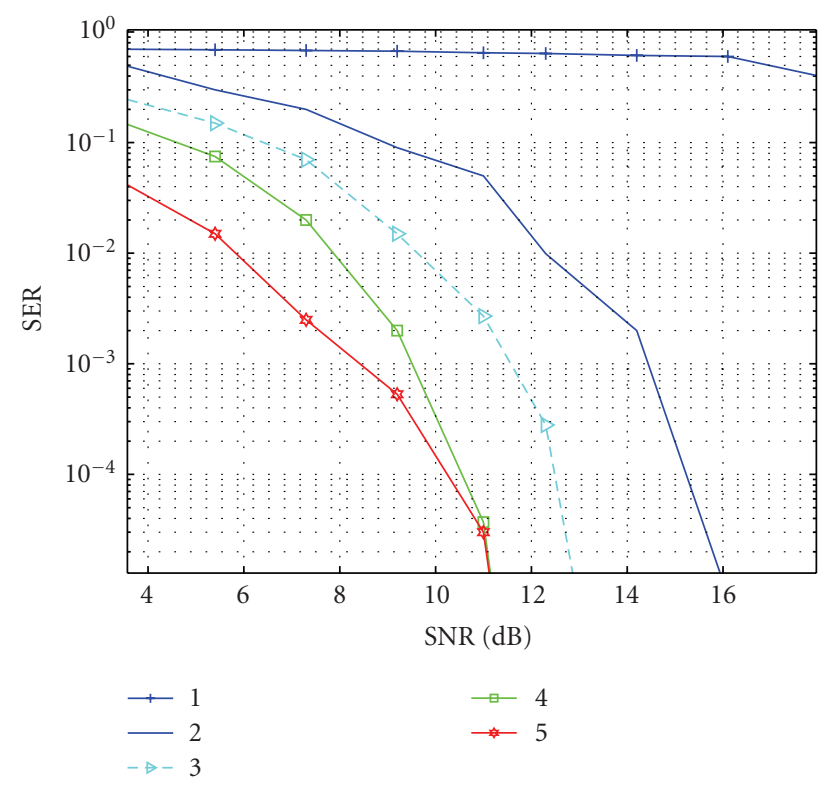

FIGURE 5: SER comparison for different equalizers. (1) Proposed algorithm without improvements discussed in Section 6, (2) LópezValcarce's Method in [11] without denoising. (3) López-Valcarce's method in [11] with matrix denoising. (4) Improved proposed algorithm by the POR $(m=3)$ method, (5) Improved proposed method by the hybrid $(m=2)$ method.

The $\left\{b_{n}\right\}$ is the input stream of $i d$ bits, that is, $b_{n} \in\{1,0\}$. The order of all equalizers is 12 , and the number of delays equals 5. Figure 5 shows the average curve of 20 runs for the relationship between the SNR and the symbol error rate (SER) relationship of the López-Valcarce algorithm and our proposed algorithms.

From Figure 5, we can see that without the improvement techniques discussed in Section 6 our proposed algorithm does not generate satisfactory equalization. Nevertheless, our improved algorithms based on the POR (with $m=$ 3) method and the hybrid (with $m=2$ ) methods outperform the López-Valcarce algorithm and even the López-Valcarce algorithm with denoised autocorrelation estimation. However, the López-Valcarce algorithm with autocorrelation matrix denoising usually requires three singular value decompositions (SVD) of matrixes with size of $M p \times M p$. To the contrary, our proposed algorithm with POR improvement only requires one SVD to find the minimum eigenvector. Furthermore, based on our proposed new constraints, an adaptive blind channel equalization method for a linear channel with colored input can be straightforwardly developed using the same approach in [15]. Consequently, we believe that our proposed algorithm combined with the POR technique provides an excellent solution for blind equalization of a linear channel with colored input in term of performance and computational complexity. We also conducted the same simulation but on randomly generated channels, which generate the similar relationship. 


\section{CONCLUSIONS}

A new direct blind linear system equalization method has been developed based on the constrained optimum method. The resulting algorithm extracts channel information from the noise subspace. However, unlike the previous TX's CMOV, our proposed algorithm is guaranteed to work for either white or colored inputs with performance close to that of the MMSE equalizer with high SNR input. The new algorithm can be regarded as an extension of the CMOV algorithm developed by Tsatsanis and Xu. Several methods are introduced to improve the performance of the introduced algorithm. Simulation results confirm the effectiveness of our developed algorithms and analyses.

\section{REFERENCES}

[1] Z. Ding and L. Ye, Blind Equalization and Identification, Marcel Dekker, New York, NY, USA, 2001.

[2] K. Abed-Meraim, W. Qiu, and Y. Hua, "Blind system identification," Proceedings of the IEEE, vol. 85, no. 8, pp. 1310-1322, 1997.

[3] L. Tong, G. Xu, and T. Kailath, "Blind identification and equalization based on second-order statistics: a time domain approach," IEEE Transactions on Information Theory, vol. 40, no. 2, pp. 340-349, 1994.

[4] H. Liu, G. Xu, and L. Tong, "A deterministic approach to blind identification of multi-channel FIR systems," in Proceedings of IEEE International Conference on Acoustics, Speech, and Signal Processing (ICASSP '94), vol. 4, pp. 581-584, Adelaide, Australia, April 1994.

[5] E. Moulines, P. Duhamel, J.-F. Cardoso, and S. Mayrargue, "Subspace methods for the blind identification of multichannel FIR filters," IEEE Transactions on Signal Processing, vol. 43, no. 2, pp. 516-525, 1995.

[6] K. Abed Meraim, P. Duhamel, D. Gesbert, et al., "Prediction error methods for time-domain blind identification of multichannel FIR filters," in Proceedings of the 20th IEEE International Conference on Acoustics, Speech, and Signal Processing (ICASSP '95), vol. 3, pp. 1968-1971, Detroit, Mich, USA, May 1995.

[7] D. T. M. Slock, "Blind fractionally-spaced equalization, perfect-reconstruction filter banks and multichannel linear prediction," in Proceedings of IEEE International Conference on Acoustics, Speech, and Signal Processing (ICASSP '94), vol. 4, pp. 585-588, Adelaide, Australia, April 1994.

[8] Q. Zhao and L. Tong, "Adaptive blind channel estimation by least squares smoothing," IEEE Transactions on Signal Processing, vol. 47, no. 11, pp. 3000-3012, 1999.

[9] Z. Ding, "Blind channel identification algorithm based on matrix outer-product," in Proceedings of IEEE International Conference on Communications (ICC '96), vol. 2, pp. 852-856, Dallas, Tex, USA, June 1996.

[10] Z. Ding, "Matrix outer-product decomposition method for blind multiple channel identification," IEEE Transactions on Signal Processing, vol. 45, no. 12, pp. 3053-3061, 1997.

[11] R. López-Valcarce and S. Dasgupta, "Blind channel equalization with colored sources based on second-order statistics: a linear prediction approach," IEEE Transactions on Signal Processing, vol. 49, no. 9, pp. 2050-2059, 2001.

[12] K. H. Afkhamie and Z.-Q. Luo, "Blind identification of FIR systems driven by Markov-like input signals," IEEE
Transactions on Signal Processing, vol. 48, no. 6, pp. 1726-1736, 2000.

[13] M. K. Tsatsanis and Z. Xu, "Constrained optimization methods for direct blind equalization," IEEE Journal on Selected Areas in Communications, vol. 17, no. 3, pp. 424-433, 1999.

[14] Z. Xu, P. Liu, and X. Wang, "Towards closing the gap between MOE and subspace methods," in Proceedings of the 36th Asilomar Conference on Signals Systems and Computers, vol. 1, pp. 689-693, Pacific Groove, Calif, USA, November 2002.

[15] Z. D. Xu and M. K. Tsatsanis, "Adaptive minimum variance methods for direct blind multichannel equalization," Signal Processing, vol. 73, no. 1-2, pp. 125-138, 1999.

[16] J. Mannerkoski and V. Koivunen, "Autocorrelation properties of channel encoded sequences-applicability to blind equalization," IEEE Transactions on Signal Processing, vol. 48, no. 12, pp. 3501-3507, 2000.

[17] D. Zhou and V. DeBrunner, "Blind channel equalization with colored source based on constrained optimization methods," in Proceedings of IEEE Global Telecommunications Conference (GLOBECOM '04), vol. 4, pp. 2286-2291, Dallas, Tex, USA, November-December 2004.

[18] S. Haykin, Adaptive Filter Theory, Prentice Hall, Upper Saddle River, NJ, USA, 4th edition, 2004.

[19] J. Capon, "High-resolution frequency-wavenumber spectrum analysis," Proceedings of the IEEE, vol. 57, no. 8, pp. 1408-1418, 1969.

[20] L. Ljung, System Identification, Prentice Hall, Upper Saddle River, NJ, USA, 2nd edition, 2002.

[21] D. H. Johnson and D. E. Dudgeon, Array Signal Processing: Concepts and Techniques, Prentice-Hall, Englewood Cliffs, NJ, USA, 1993.

[22] Z. Xu, P. Liu, and X. Wang, "Blind multiuser detection: from MOE to subspace methods," IEEE Transactions on Signal Processing, vol. 52, no. 2, pp. 510-524, 2004. 